ARTICLE

\title{
Site-controlled telecom-wavelength single-photon emitters in atomically-thin $\mathrm{MoTe}_{2}$
}

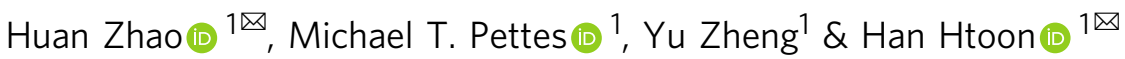

Quantum emitters (QEs) in two-dimensional transition metal dichalcogenides (2D TMDCs) have advanced to the forefront of quantum communication and transduction research. To date, QEs capable of operating in O-C telecommunication bands have not been demonstrated in TMDCs. Here we report site-controlled creation of telecom QEs emitting over the 1080 to $1550 \mathrm{~nm}$ telecommunication wavelength range via coupling of 2D molybdenum ditelluride $\left(\mathrm{MoTe}_{2}\right)$ to strain inducing nano-pillar arrays. Hanbury Brown and Twiss experiments conducted at $10 \mathrm{~K}$ reveal clear photon antibunching with $90 \%$ single-photon purity. The photon antibunching can be observed up to liquid nitrogen temperature (77 K). Polarization analysis further reveals that while some QEs display cross-linearly polarized doublets with $\sim 1 \mathrm{meV}$ splitting resulting from the strain induced anisotropic exchange interaction, valley degeneracy is preserved in other QEs. Valley Zeeman splitting as well as restoring of valley symmetry in cross-polarized doublets are observed under $8 \mathrm{~T}$ magnetic field.

\footnotetext{
${ }^{1}$ Center for Integrated Nanotechnologies, Materials Physics and Applications Division, Los Alamos National Laboratory, Los Alamos, New Mexico 87545,

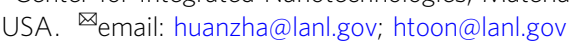


uantum emitters (QEs) that emit one photon at a time are key building blocks for numerous quantum technology protocols such as quantum communications, quantum information processing, and quantum key distribution ${ }^{1-4}$. In particular, QEs operating in the telecom bands $(1.25-1.55 \mu \mathrm{m})$ are highly desired for implementation of quantum technologies through existing fiber-based optical communication networks. Currently, telecom-compatible QEs have been demonstrated in various III-V semiconductor quantum dots ${ }^{5,6}$ and recently in functionalized carbon nanotubes ${ }^{7}$. However, several challenges such as accurate site positioning and efficient polarization control still remain for these QEs. Over the past decade, two-dimensional (2D) semiconductors have emerged as a novel platform for both fundamental research and technological applications. Leveraged by the unique membrane-like geometry, 2D semiconductors are promising for QEs, as they offer high photon extraction efficiency, easy coupling to external fields, and convenient integration with photonic circuits. In addition, strain engineering can be applied to accurately position the emission $\operatorname{sites}^{8-11}$. Most importantly, 2D transition metal dichalcogenides (TMDCs) have a valley degree of freedom that can be manipulated and accessed through circularly polarized excitonic optical transitions and efficiently tuned via a magnetic field ${ }^{12,13}$, bringing new quantum functionalities to embedded QEs. Recently, 2D QEs have been demonstrated in $\mathrm{WSe}_{2}{ }^{14-17}$ and hexagonal boron nitride ${ }^{18,19}$, covering a broad emission spectrum range from $\sim 500$ to $800 \mathrm{~nm}$. However, single-photon emission in the most desirable spectral range-the telecom bands-has never been explored in 2D systems. Although most 2D semiconductors have inherent electronic band structures that limit the operating wavelength to the visible range, $\alpha$-phase $2 \mathrm{H}$-molybdenum ditelluride $\left(\mathrm{MoTe}_{2}\right)$ has a layerdependent bandgap in the NIR regime, holding promise for telecom-compatible single-photon emission. However, extending the nanoscale strain engineering approach to $\mathrm{MoTe}_{2}$ has been a major technical challenge. Although QEs can even form naturally ${ }^{14-17}$ and can be induced with almost any type of nanoscale topological features starting from bubbles ${ }^{20}$ to lithographically defined patterns ${ }^{8-11}$ in $\mathrm{WSe}_{2}$, no natural QEs have been reported in $\mathrm{MoTe}_{2}$ and whether $\mathrm{MoTe}_{2}$ could host QEs remains unexplored.

Here we report site-controlled telecom single-photon emission in $\mathrm{MoTe}_{2}$ mono- and few layers by transferring mechanically exfoliated $\mathrm{MoTe}_{2}$ thin flakes onto strain-inducing nano-pillar arrays. We observed QEs in both monolayer and relatively thick (>10 layers) $\mathrm{MoTe}_{2}$ flakes, with emission wavelength covering the entire near-infrared (NIR) emission band from $1080 \mathrm{~nm}$ to $1550 \mathrm{~nm}$. Our Hanbury Brown and Twiss (HBT) experiment yielded near-complete photon antibunching, unambiguously proving the single-photon nature of the emitters. Polarizationresolved magneto-optical spectroscopies further revealed that although valley symmetry is preserved in some QEs, the straininduced anisotropic exchange interaction can mix valley states in other QEs to display cross-linearly polarized doublets with $\sim 1 \mathrm{meV}$ splitting at zero magnetic field. The valley symmetry in these QEs is restored under $8 \mathrm{~T}$ magnetic field.

\section{Results}

Near-band-edge QEs in monolayer $\mathrm{MoTe}_{2}$. Figure 1a presents an optical image of a $\mathrm{MoTe}_{2}$ monolayer on a nano-pillar array. The sample preparation and nanofabrication processes are discussed in "Methods" section and Supplementary Note 1. The corresponding wide-field photoluminescence (PL) image (Fig. 1b) shows significantly brighter PL emission in the regions directly contacting the nano-pillars. Our initial efforts using naturally formed bubbles and lithographically defined Si nanostructures to induce strain in $\mathrm{MoTe}_{2}$ only produced broad featureless emissions (Supplementary Fig. 3). Unstrained monolayer $\mathrm{MoTe}_{2}$ features two dominant emission peaks at cryogenic temperature: exciton $\left(X^{0}\right)$ emission at around $1050 \mathrm{~nm}$ and trion $\left(X^{ \pm}\right)$emission at around $1070 \mathrm{~nm}$ (Fig. 1c, lower panel) ${ }^{21,22}$. With localized strain, a series of narrow PL peaks covering a broad spectral range of 1080-1150 nm emerges from the lower-energy side of the spectrum (Fig. 1c upper panel and Supplementary Fig. 4). A single nano-pillar often produces multiple sharp PL peaks at slightly different spatial locations (determined through the centroid of the PL emission). Following recent theoretical studies, we attribute these PL peaks to the localized exciton states arising from strain-induced hybridization of dark excitons and the defect states $^{23,24}$. As our nano-pillars induce deformation over several hundreds of nanometers (Supplementary Fig. 2) and are thus capable of hosting multiple point defects, spatially distinct localized exciton states could form in a single strained region. An increase in PL intensity of trion $\left(X^{ \pm}\right)$emission relative to that of $X^{0}$ emission in the strained region further indicates that the redistribution of free carriers under non-uniform strain profiles observed previously in $\mathrm{WS}_{2}{ }^{25}$ could also occur in our case and localized exciton states could also be charged. The linewidths of the QE PL emission range from a few meV to sub-meV at $4-10 \mathrm{~K}$ temperatures, which are nearly an order of magnitude narrower than the linewidths of the $2 \mathrm{D}$ exciton PL peaks $(\sim 10 \mathrm{meV})$. Supplementary Fig. 5 displays a narrower PL peak of $0.6 \mathrm{meV}$ full width at half maximum (FWHM) observed at $4 \mathrm{~K}$. These narrow, near-band-edge emission lines are also frequently observed in two to four layered $\mathrm{MoTe}_{2}$ but are not seen in much thicker flakes. Figure $1 \mathrm{~d}$ shows a typical narrow PL peak from a QE that displays a FWHM of $920 \mu \mathrm{eV}$. The peak is accompanied by a weak shoulder peak at $\sim 2.5 \mathrm{meV}$ higher energy. Both the width and shape of this PL peak remain essentially unchanged over nearly three orders of magnitude change in pump-power. The PL intensity also varied linearly with pump-power and only showed weak saturation at powers $>300 \mathrm{nW}$ (Fig. 1e).

To access the single-photon-emission characteristics of the QEs, we performed time-tagged, time-correlated single-photon counting and HBT experiments. The PL intensity time trace, PL decay curve, and second-order correlation at zero time delay, or $g^{2}(0)$, was extracted from the photon stream. Figure 2a displays the PL spectrum of a localized emitter, of which the measurement results are presented in Fig. 2b-d. The PL decay curve (Fig. 2b) shows a near-perfect single-exponential decay with a lifetime of $\tau=22.2 \pm 0.1 \mathrm{~ns}$, four orders of magnitude longer than that in pristine $\mathrm{MoTe}_{2}(\sim 2 \mathrm{ps})^{26}$. This long lifetime provides a clear indication that localization of the exciton in a strain-induced potential trap suppresses non-radiative recombination through defect states that dominate the decay of 2D band-edge excitons.

Quantum-dot-like solid-state QEs typically have instability issues such as photobleaching, blinking, and spectral diffusionall of which hinder applications ${ }^{27}$. We monitored the timedependent PL emission under both pulses and continuous wave (CW) laser excitation (Fig. 2b inset and Supplementary Fig. 6), revealing stable emission at an average count rate of 1.2 and $9 \mathrm{kHz}$ for pulsed and $\mathrm{CW}$ excitation, respectively, without detectable photobleaching or blinking over the timescales presented. A time series of PL spectra with $0.4 \mathrm{meV}$ spectral resolution also show no spectral diffusion (Supplementary Fig. 7). Figure $2 \mathrm{c}$, d present the photon correlation under pulsed $\left[g^{(2)}\right.$ $(0)=0.058 \pm 0.003]$ and CW $\left[g^{(2)}(0)=0.181 \pm 0.030\right]$ excitation, respectively. Both values are well below the photon antibunching threshold of $g^{(2)}(0)=0.5$, which unequivocally reveals that the strain-induced $\mathrm{MoTe}_{2}$ localized emitter is indeed a QE. HBT experiments conducted on a different emitter at 40 and $77 \mathrm{~K}$ prove that the single-photon-emission behavior can survive up to 
a
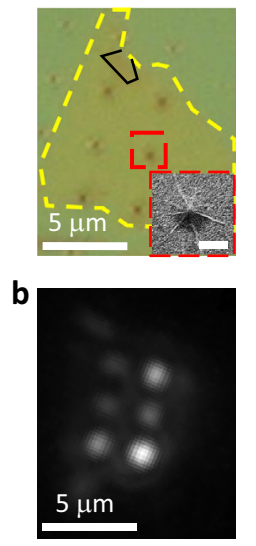

C

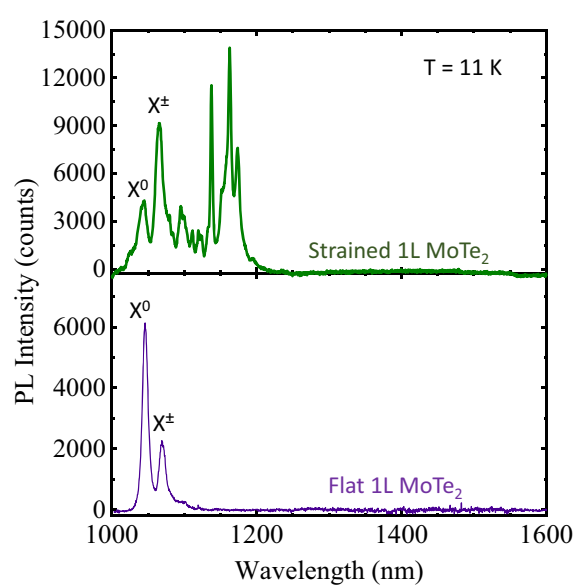

d

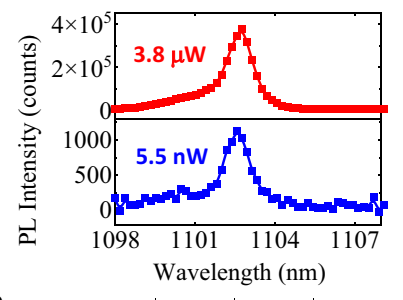

e

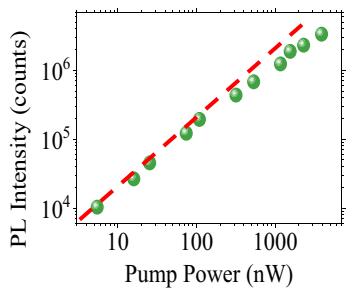

Fig. 1 Near-band-edge sharp emission lines obtained from strained monolayer $\mathbf{M o T e}_{\mathbf{2}}$ - a Optical image of a monolayer MoTe $\mathbf{f}_{2}$ flake (with a tiny folded bilayer region outlined in black) on a nano-pillar array. The MoTe $e_{2}$ flake is outlined by yellow dashed lines. Scale bar: $5 \mu$ m. Inset: SEM image of a nanopillar coated with monolayer MoTe $\mathrm{N}_{2}$ displaying a tent-shaped profile. Scale bar of the inset: $500 \mathrm{~nm}$. b Wide-field PL image of the same flake. The brighter areas indicate stronger emission and are consistent with the pillar locations in Fig. 1a. Scale bar: $5 \mu \mathrm{m}$. c PL spectrum of a MoTe 2 monolayer on flat PMMA (lower panel) and the PL spectrum of a MoTe $\mathrm{M}_{2}$ monolayer sitting on a nano-pillar (upper panel) acquired at laser excitation power of $0.8 \mu \mathrm{W}$ and $30 \mathrm{~s}$ integration time. The MoTe $\mathrm{M}_{2}$ exciton and trion peaks are identified. $\mathbf{d} \mathrm{PL}$ spectra of a representative localized MoTe $\mathrm{e}_{2}$ emitter acquired at $3.3 \mu \mathrm{W}$ and $5.5 \mathrm{nW}$ pump-power showing invariant shape and width ( $920 \mu \mathrm{eV}$ at FWHM) of the spectral line. e Pump-power-dependent emission intensity of the PL peak presented in Fig. 1d shows near-perfect linear scaling with pump-power. The red dashed line is a guide to the eye. All data were obtained at $11 \mathrm{~K}$ temperature.

a
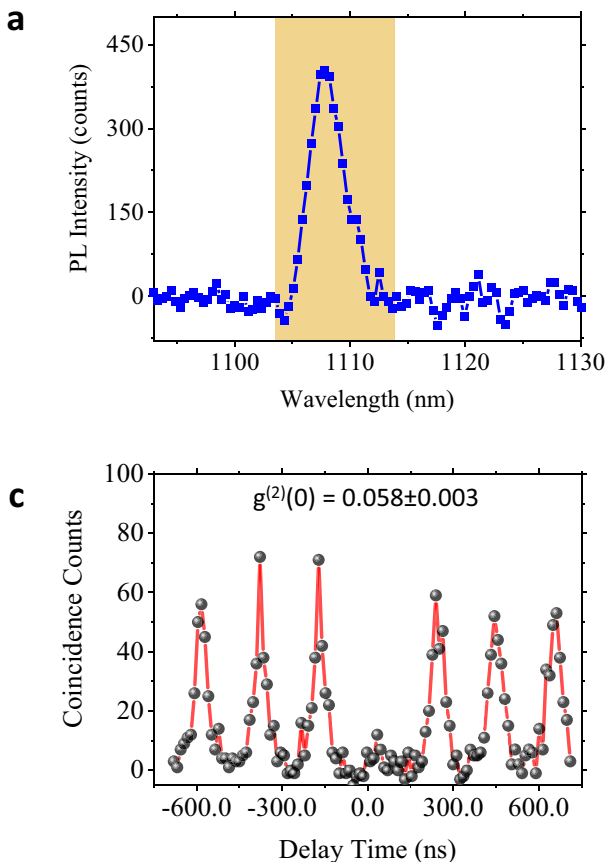

b
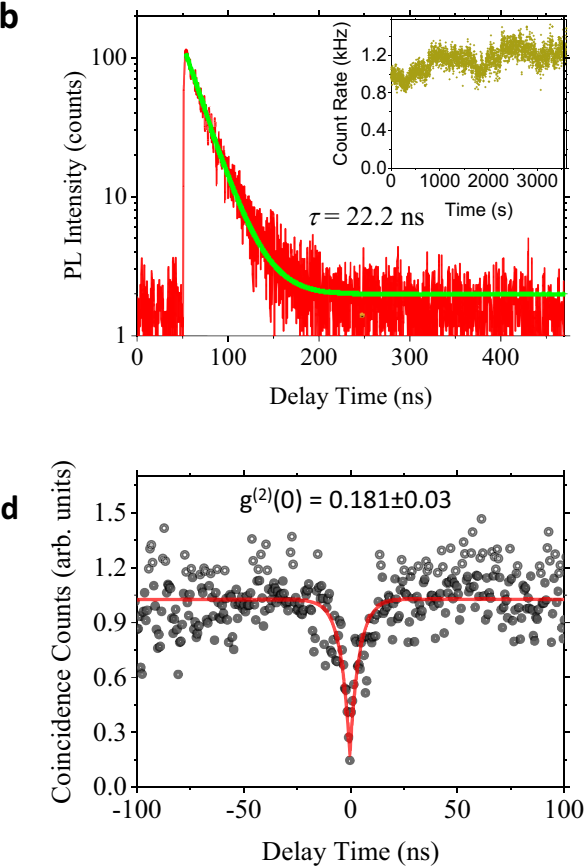

Fig. 2 PL dynamics of a near-band-edge MoTe2 QE. a PL spectrum of a localized MoTe $e_{2}$ emitter. The data of Fig. 2b-d are taken from this QE with a bandpass filter that allows the shadowed region to be detected. $\mathbf{b}$ The PL decay curve (red) and a single-exponential decay fit (green) with a $22.2 \pm 0.1$ ns extracted lifetime. Inset: PL intensity as a function of experiment time showing stability of the single-photon emission rate over $1 \mathrm{~h}$ time, the modulation observed over the long timescale $(\sim 100 \mathrm{~s})$ is mainly due to sample drift relative to the laser excitation focal spot. c Second-order correlation measurement under $850 \mathrm{~nm}$ pulsed excitation with a $2.1 \mathrm{MHz}$ repetition rate, from which a $g^{(2)}(0)=0.058 \pm 0.003$ is extracted. $\mathbf{d}$ Second-order correlation measurement under $850 \mathrm{~nm}$ CW laser excitation. The red curve is a fit to the data using a bi-exponential decay function. The extracted $g^{(2)}(0)$ of $0.181 \pm 0.030$ is slightly higher than the pulsed measurement due to higher Poissonian background caused by CW excitation. All data were obtained at $11 \mathrm{~K}$ temperature. 


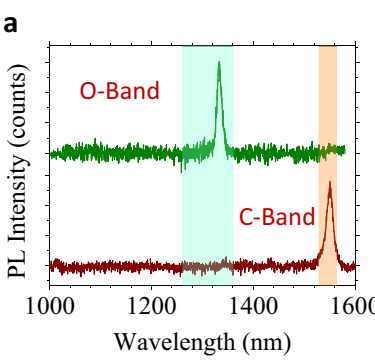

d

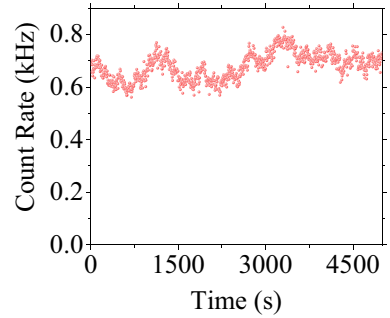

b

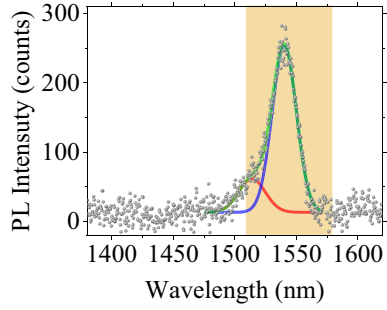

e

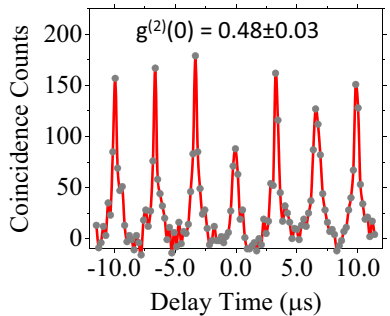

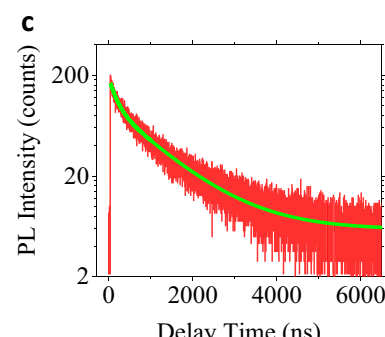

f

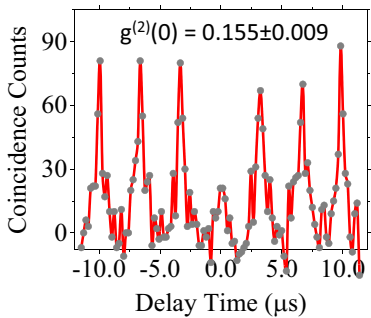

Fig. 3 PL spectra and PL dynamics of telecom QEs. a "O-band" and "C-band" telecom PL emissions from two multilayer MoTe $\mathrm{H}_{2}$ localized emitters, corresponding telecom bands indicated by shaded regions. b MoTe 2 QE with a $1540 \mathrm{~nm}$ telecom emission peak and a small shoulder peak at $1510 \mathrm{~nm}$. The blue and red lines are Gaussian fits to the 1540 and $1510 \mathrm{~nm}$ peaks, respectively. The data of Fig. 3c-f are taken from this emitter with a band-pass filter that allows only the shaded region to be detected. c PL decay curve (red) and bi-exponential decay fit (green) reveal the lifetime of the $1540 \mathrm{~nm}$ peak is $1.13 \pm 0.01 \mu \mathrm{s}$. d Time-dependent PL counts showing stable emission over $5000 \mathrm{~s}$. Data are recorded every $500 \mathrm{~ms}$. e Second-order correlation measurement under $850 \mathrm{~nm}$ pulsed excitation with a $330 \mathrm{kHz}$ repetition rate, from which $g^{(2)}(0)=0.48 \pm 0.03$ is extracted. $\mathbf{f}$ Time-gated $g^{(2)}$ shows $g^{(2)}$

$(0)=0.155 \pm 0.009$ under a $200 \mathrm{~ns}$ time gate. All data were obtained at $13 \mathrm{~K}$ temperature.

liquid nitrogen temperature, although the emission intensity decreases significantly due to thermally activated non-radiative recombination (Supplementary Fig. 8). Normalizing the measured count rates of the QE of Fig. 2 with the overall collection efficiency of our micro-PL system at $1108 \mathrm{~nm}(0.035 \%$, see Supplementary Note 4 ), we estimate the corrected emission rate of the QE to be 3.4 and $25.6 \mathrm{MHz}$ for pulsed and $\mathrm{CW}$ excitation, respectively. Assuming that an exciton is localized into the QE in every excitation cycle, we can further deduce the radiative recombination efficiency of the $\mathrm{QE}$ to be $\sim 7 \%$ from the ratio between the corrected emission rate under pulsed excitation and the $48.5 \mathrm{MHz}$ pulsed excitation rate $^{8,28,29}$.

Telecom-wavelength QEs in multilayer $\mathbf{M o T e}_{2}$. Obtaining telecom-compatible QEs that emit at around $1.3 \mu \mathrm{m}$ (O-band, "original band") and $1.55 \mu \mathrm{m}$ (C-band, "conventional band") are required for fiber-based quantum communications. When increasing the $\mathrm{MoTe}_{2}$ layer numbers from monolayer to bulk, the bandgap of $\mathrm{MoTe}_{2}$ monotonically decreases from $1.18 \mathrm{eV}(1050 \mathrm{~nm})$ to $0.95 \mathrm{eV}$ $(1300 \mathrm{~nm})^{22}$. The PL intensity also decreases by orders of magnitude due to a direct-to-indirect bandgap transition commonly observed in 2D TMDCs. In our experiment, we observe bright telecom-band emission created from strained few-layer $\mathrm{MoTe}_{2}$, although we have occasionally found such emissions in mono- and bilayer samples (Supplementary Notes 7-9). Figure 3a and Supplementary Fig. 9 present PL spectra of telecom-wavelength emitters. We typically observe such highly red-shifted bright emissions spanning $1.25-1.55 \mu \mathrm{m}$, covering the full telecom window. Supplementary Fig. 10 demonstrates that QEs emitting at wavelengths longer than $1300 \mathrm{~nm}$ are created in five- to six-layer $\mathrm{MoTe}_{2}$ at almost all nanopillar sites. In contrast to near-band-edge QEs, these QEs are characterized by relatively broad linewidths (FWHM $7 \sim 30 \mathrm{meV}$ ).

Figure $3 \mathrm{~b}$ presents the PL spectrum of a telecom QE, for which the PL dynamics and photon correlation results are presented in Fig. 3c-f. We observed an initial PL decay with a lifetime of $163 \pm 3 \mathrm{~ns}$, followed by an ultra-long lifetime of $1.13 \pm 0.01 \mu \mathrm{s}$.
This ultra-long lifetime is attributed to the dominant PL peak at lower energy, confirmed by comparing the integrated PL counts from the PL decay curve with the PL counts in the spectrum (see Supplementary Note 10). The measured lifetime is six orders of magnitude longer than that of the $\mathrm{MoTe}_{2}$ band-edge emission and is two to three orders longer than that of our near-band-edge QEs. Based on this long lifetime and the fact that these telecom QEs are observed more frequently in multilayer $\mathrm{MoTe}_{2}$, we attribute the telecom QEs to indirect excitonic transitions, which are activated by strain-induced quantum confinement potentials. Figure 3d shows that this telecom QE is also free from blinking or photobleaching over a $5000 \mathrm{~s}$ time period. The HBT experiment under pulsed excitation for the spectral window shown in Fig. $3 \mathrm{~b}$ yields $g^{(2)}(0)=0.48 \pm 0.03$ (Fig. 3e). As this measurement includes contribution from a high-energy shoulder that exhibits a shorter PL decay time constant, we employed a time-gated $g^{(2)}$ experiment $^{30}$ (Supplementary Note 11), in which only the photons arriving after the decay of the higher energy shoulder (i.e., after 200 ns delay) were analyzed for the $g^{(2)}$ trace. The timegated $g^{(2)}$ in Fig. 3f shows $g^{(2)}(0)=0.155 \pm 0.009$, clearly proving the antibunching nature of the $1540 \mathrm{~nm}$ telecom emitter. HBT experiments of two other QEs emitting at 1340 (O-band) and $1233 \mathrm{~nm}$ yielded $g^{(2)}(0)$ of $0.28 \pm 0.02$ and $0.10 \pm 0.01$, respectively, and are shown in Supplementary Fig. 11. Using the overall collection efficiency of our micro-PL system at $1550 \mathrm{~nm}(0.31 \%)$ (Supplementary Note 4) and $303 \mathrm{kHz}$ pulsed excitation rate, we can estimate the absolute emission rate and radiative recombination efficiency of the QE shown in Fig. 3 to be $258 \mathrm{kHz}$ and $85 \%$, respectively $8,28,29$.

Polarization-resolved magneto-optical spectroscopies. To investigate the valley physics of QEs in $\mathrm{MoTe}_{2}$, we conducted polarizationresolved magneto-PL spectroscopy with magnetic field normal to the sample surface (Faraday geometry). Figure 4a presents helicityresolved PL of a MoTe 2 QE. The spectra was taken with $\sigma^{+}$excitation and analyzed for both $\sigma^{+}$and $\sigma^{-}$helicities. The valley Zeeman 
a

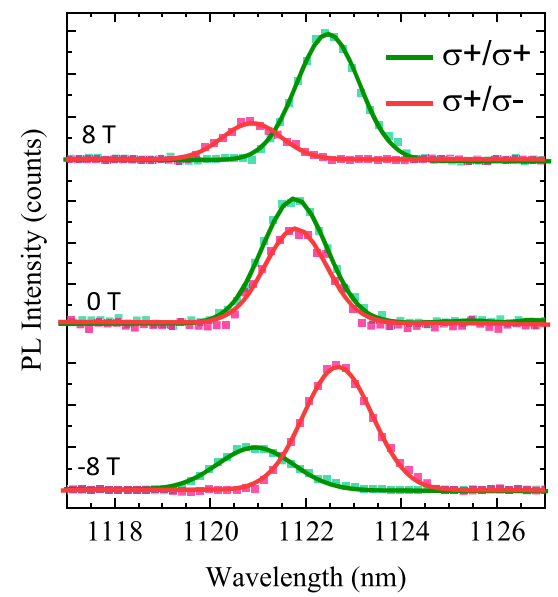

C

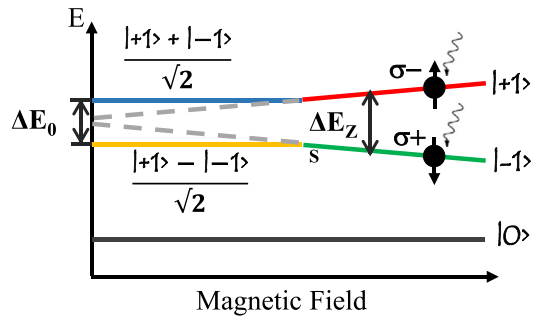

b

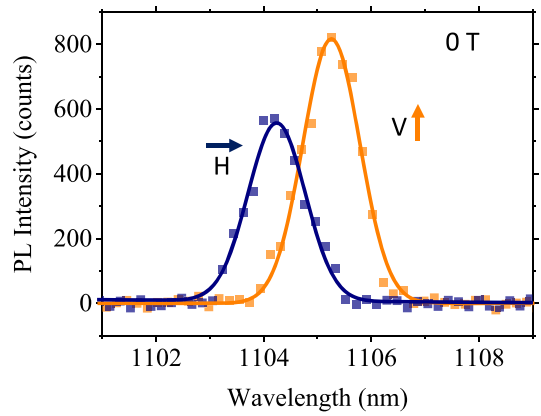

d

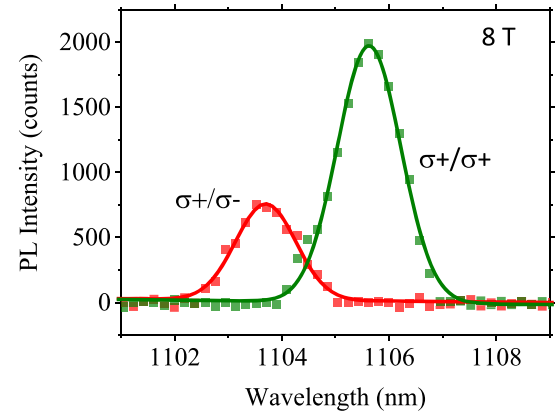

Fig. 4 Polarization-resolved magneto-optical spectroscopies. a Helicity-resolved magneto-PL data of a MoTe ${ }_{2}$ QE. The emitter was excited using a $\sigma^{+}$ polarized laser. $\mathbf{b}$ The PL spectrum of a linearly cross-polarized doublet measured at zero field, showing a fine-structure energy splitting of $\sim 1.1 \mathrm{meV}$. $\mathrm{H}$ and $\checkmark$ denote horizontal and vertical polarization detection directions, respectively. c Energy diagram of the doublet as a function of the external magnetic field. The linearly polarized states (yellow and blue lines) are converted into $\sigma^{+}$and $\sigma^{-}$circularly polarized states (green and red lines) once the Zeeman energy $\left(\Delta E_{Z}\right)$ exceeds the zero-field fine-structure splitting energy $\left(\Delta E_{0}\right)$. The Zeeman splitting energy at linear polarization state is indicated by the gray dash lines. The ground state is denoted as $|0\rangle$. All data were obtained at $11 \mathrm{~K}$ temperature. $\mathbf{d}$ Spectrum of the same doublet under an $8 \mathrm{~T}$ magnetic field, showing the doublet is converted to a circularly cross-polarized pair. The excitation is a $\sigma^{+}$polarized laser. Lines in Fig. $4 a-c$ are Gaussian peak fits to the PL data.

splitting was not observed in the absence of a magnetic field but rose with increasing field, indicating a lifting of valley degeneracy. Using the relation between the energy splitting $\Delta E$ and the magnetic field $\mathbf{B}$, $\Delta E=-g \mu_{\mathrm{B}} \mathbf{B}$, where $\mu_{\mathrm{B}}$ is the Bohr magneton, we extracted a Landé $g$-factor of $-3.61 \pm 0.02$ for the QE (Supplementary Note 12), which is comparable with $g$-factors reported in other work ${ }^{31}$. At zero field, the degree of circular polarization, $P_{\mathrm{C}}=\left(I_{\sigma+}-I_{\sigma-}\right) /\left(I_{\sigma+}+I_{\sigma-}\right)$ was $13 \%$ as a result of the valley-selective pumping effect. Significant valley polarization was observed with an applied magnetic field, reaching $52 \%$ at $8 \mathrm{~T}$, which is more pronounced compared to the $30 \%$ polarization reported in magnetic-field-induced valley polarization of intrinsic $\mathrm{MoTe}_{2}$ excitons ${ }^{31}$. These results further indicate a vanishing fine-structure splitting of some of the QEs at zero field that could arise from azimuthally symmetric strain-induced confinement potentials $^{32}$. In this case, these QEs hold great potential for entangled photon generation ${ }^{33}$. However, vanishing fine-structure splitting could also result from charging of the $\mathrm{QE}^{34,35}$. As the g-factor of neutral and charged excitons of $\mathrm{MoTe}_{2}$ are almost identical $(-4.6 \pm 0.2 \text { for exciton and }-4.5 \pm 0.3 \text { for trion })^{31}$, our magneto-PL experiment cannot be used to distinguish these two possibilities, similar to the case of $\mathrm{WSe}_{2} \mathrm{QEs}^{16,32,34,35}$.

We also observed emission pairs in some of the QEs, which were found to be cross-linearly polarized doublets with sizeable zero-field energy splitting (1-3.7 meV) (Fig. 4b and Supplementary Note 13). The two peaks from the doublet have a zero-field splitting of $1.09 \mathrm{meV}$ and reach their maximal PL intensities in opposite linear polarization directions (horizontal/vertical), indicating the presence of fine structure. The observed crosspolarization and zero-field energy splitting have also been reported in III-V quantum dots ${ }^{36,37}$ and recently in $\mathrm{WSe}_{2}$ $\mathrm{QEs}^{15,17}$. The higher PL intensity of the lower-energy state is attributed to the efficient thermal relaxation process that has also been reported in $\mathrm{WSe}_{2} \mathrm{QEs}^{15,17}$. Following prior studies, we attribute this fine-structure splitting to hybridization of $\mathrm{K}$ and $\mathrm{K}$ ' valley-polarized excitons by an asymmetric potential landscape defined by the localized strain as illustrated in Fig. 4c. When the magnetic field is strong enough to overcome the anisotropic Coulomb potential (i.e., $\Delta E_{\mathrm{Z}}>\Delta E_{0}$ ), the linearly polarized states vanish and circularly polarized states are recovered. This restoration of valley symmetry is achieved in our QE under an $8 \mathrm{~T}$ magnetic field (Fig. $4 \mathrm{~d}$ ). Compared to the $\sim 0.7 \mathrm{meV}$ finestructure splitting energy of as-exfoliated $\mathrm{WSe}_{2} \mathrm{QEs}^{15,17}$, the larger zero-field splitting energy in our $\mathrm{MoTe}_{2}$ QEs suggests that the nano-pillar-based strain engineering technique can induce more confining potential anisotropy compared to native strain.

\section{Discussion}

In this work, we report site-controlled creation of two types of QEs using nano-pillar-based strain engineering: near-band-edge QEs covering 1080-1150 nm in mono- and few-layer $\mathrm{MoTe}_{2}$ and telecom QEs emitting in 1200-1600 nm range in multilayer $\mathrm{MoTe}_{2}$. The quantum natures of the QEs were verified by photon correlation measurements up to liquid nitrogen temperature. Compared to telecom QEs, the near-band-edge QEs has a relatively low radiative recombination efficiency, which could result from thermal hopping of excitons out of the shallow trapping potential. This efficiency increases significantly when the QE is isolated in energy from the band-edge, as demonstrated in our telecom QEs. The deep-trapping 
potential of the telecom QEs strongly suppresses thermal de-trapping of excitons, which is believed to be responsible for the low radiative efficiency of band-edge QEs. These results indicate that we can utilize the thickness of the $\mathrm{MoTe}_{2}$ layer as a control knob for the creation of two types of QEs. In contrast, only near-band-edge QEs can be created in $\mathrm{WSe}_{2}{ }^{8,14-17,34,35,38}$ and only deep $(110-220 \mathrm{meV})$ trap states with long lifetime can be created in $\mathrm{MoS}_{2}{ }^{39}$. Although the long PL lifetime and broad emission linewidth of telecom QEs are generally not desirable as they could limit generation rate and indistinguishability of single photons, recent studies have shown that both of these limitations can be overcome by exploiting plasmonic enhancement ${ }^{8,40}$. As in the case of all $2 \mathrm{D}$ QEs, control over the emission wavelength remains a major challenge. In addition, we observed cross-polarized doublets with $\sim 1.1 \mathrm{meV}$ zero-field energy splitting in some of the localized emitters, suggesting a strong anisotropic confinement. The polarization state of the QEs was found to be tunable by external magnetic field. Our findings extend the operating wavelength of $2 \mathrm{D}$ QEs into the NIR regime, bringing in solutions for creating site-controlled stable telecom-compatible QEs. We envisage various future directions inspired by our early-stage demonstration, including electrically driven telecom QEs and cavityenhanced tunable NIR QEs. We are also encouraged by the possibility of realizing room-temperature $\mathrm{MoTe}_{2}$ telecom QEs, as the energy redshift between the telecom emission and the $\mathrm{MoTe}_{2}$ exciton emission is well above the thermal energy at room temperature. Finally, an in-depth understanding of the excitonic physics of $\mathrm{MoTe}_{2}$ QEs may give rise to interesting perspectives on manipulating the spin-valley degree of freedom and designing valleytronic devices.

\section{Methods \\ Sample preparation. $\mathrm{MoTe}_{2}$ flakes were mechanically exfoliated from a flux-} grown bulk crystal before they were transferred onto pre-patterned substrates. Thin layers (i.e., flakes that look greenish and translucent under an optical microscope) were selected for further optical characterizations. One- to four-layer-thick flakes can be easily distinguished by analyzing the band-edge emission wavelength and PL intensity ${ }^{22}$. Flakes thicker than four layers do not have a detectable band-edge emission using $\mu \mathrm{W}$ level pump-power. To prepare the strained substrates, a $50 \mathrm{~nm}$ $\mathrm{Au}$ layer was deposited on top of a $\mathrm{Si} / \mathrm{SiO}_{2}$ substrate to block silicon PL emission, followed by spin-coating of a $50 \mathrm{~nm}$ polyvinyl alcohol (PVA) dielectric layer to prevent quenching effects. Then, a $\sim 120 \mathrm{~nm}$ polymethyl methacrylate (PMMA) layer was spin-coated on top of the PVA layer and patterned by electron beam lithography into PMMA nano-pillar arrays with a $3 \mu \mathrm{m}$ pitch width. Vacuum annealing at $90^{\circ} \mathrm{C}$ was performed to enhance the contact between the $2 \mathrm{D}$ flakes and the nano-pillars. Each pillar had a $\sim 100 \mathrm{~nm}$ pillar height and a $\sim 150 \mathrm{~nm}$ diameter. It is worth noting that later we found the PVA spacer layer was not necessary as the $\sim 100 \mathrm{~nm}$ PMMA pillars were sufficient to separate the $\mathrm{MoTe}_{2}$ dots from the gold layer and maintain efficient PL emission.

Optical characterization. A diagram of our optical measurement setup is presented in Supplementary Note 14. Micro-PL measurements of $\mathrm{MoTe}_{2}$ QEs were performed on a home-built confocal microscope with excitation from either an $850 \mathrm{~nm}$ CW Ti:sapphire laser or an $850 \mathrm{~nm}$ supercontinuum pulsed laser. The excitation power was typically a few $\mu \mathrm{W}$. Samples were mounted in a continuous flow cryostat and cooled to $10-13 \mathrm{~K}$ using liquid helium. The emitted light was collected through a $\times 50$ infrared objective lens (Olympus, $0.65 \mathrm{NA}$ ) and spectrally filtered before entering a 2D InGaAs array detector (NIRvana 640LN, Princeton Instruments). We used 150 and $300 \mathrm{gr} / \mathrm{mm}$ gratings to resolve the spectra. For TRPL and HBT experiments, the emission signal was spectrally filtered before coupling into a 50:50 optical fiber beamsplitter, which equivalently split the signal into two beams and sent them into two channels of a superconducting nanowire single-photon detector (Quantum Opus). PL intensity time traces, PL decay curves, and $\mathrm{g}^{(2)}$ traces were obtained from photon detection events recorded by a PicoQuant HydraHarp 400 time-correlated single-photon-counting module. We applied a bi-exponential decay model to determine the CW $g^{(2)}(0)$ value and error level. For pulsed auto-correlation measurements, $g^{(2)}(0)$ was extracted by comparing the integrated photon coincidence counts at the zero time-delay peak with the averaged integrated photon coincidence counts at 30 adjacent peaks. The error level of pulsed $g^{(2)}(0)$ was defined by the SD of the integrated photon coincidence counts in the adjacent peaks. For the pulsed $g^{(2)}(0)$ measurement of the $1540 \mathrm{~nm}$ QE peak, a time gate of $200 \mathrm{~ns}$ was applied to significantly reduce the contribution from the undesired emissions that could not be fully removed by optical filters.

For magneto spectroscopy and polarization-resolved PL measurements, the sample was placed inside the room-temperature bore of an $8.5 \mathrm{~T}$ liquid-helium cooled superconducting magnet. For linear polarization analysis, the excitation beam was fully depolarized using a laser depolarizer to eliminate the polarization memory effect. A half-wave plate (HWP) was inserted into the collection channel to rotate the polarization direction. A Wollaston prism was placed between the HWP and the InGaAs detector to spatially split the emission into horizontal and vertical components, followed by a depolarizer to avoid effects arising from the linear polarization dependence of the gratings. For circular polarization analysis, a quarter-wave plate (QWP) was inserted into the shared path of the excitation and the emission beams. As a result, the QWP turns the linearly polarized laser into a circularly polarized excitation source and converts the circularly polarized PL signals into linearly polarized beams. A HWP and a Wollaston prism were installed in the collection beam to spatially split the $\sigma^{+}$and $\sigma^{-}$emissions into two different areas on the $2 \mathrm{D}$ InGaAs array.

\section{Data availability}

The data that support the findings of this study are available from the corresponding author upon request.

Received: 10 September 2021; Accepted: 29 October 2021; Published online: 19 November 2021

\section{References}

1. Lodahl, P., Mahmoodian, S. \& Stobbe, S. Interfacing single photons and single quantum dots with photonic nanostructures. Rev. Mod. Phys. 87, 347 (2015).

2. Northup, T. E. \& Blatt, R. Quantum information transfer using photons. Nat. Photonics 8, 356-363 (2014)

3. Knill, E., Laflamme, R. \& Milburn, G. J. A scheme for efficient quantum computation with linear optics. Nature 409, 46-52 (2001).

4. Atatüre, M., Englund, D., Vamivakas, N., Lee, S.-Y. \& Wrachtrup, J. Material platforms for spin-based photonic quantum technologies. Nat. Rev. Mater. 3, 38-51 (2018).

5. Buckley, S., Rivoire, K. \& Vučković, J. Engineered quantum dot single-photon sources. Rep. Prog. Phys. 75, 126503 (2012).

6. Miyazawa, T. et al. Single-photon generation in the $1.55-\mu \mathrm{m}$ optical-fiber band from an InAs/InP quantum dot. Jpn J. Appl. Phys. 44, L620 (2005).

7. He, X. et al. Tunable room-temperature single-photon emission at telecom wavelengths from $\mathrm{sp}^{3}$ defects in carbon nanotubes. Nat. Photonics 11, 577-582 (2017).

8. Luo, Y. et al. Deterministic coupling of site-controlled quantum emitters in monolayer $\mathrm{WSe}_{2}$ to plasmonic nanocavities. Nat. Nanotechnol. 13, 1137-1142 (2018).

9. Palacios-Berraquero, C. et al. Large-scale quantum-emitter arrays in atomically thin semiconductors. Nat. Commun. 8, 15093 (2017).

10. Branny, A., Kumar, S., Proux, R. \& Gerardot, B. D. Deterministic straininduced arrays of quantum emitters in a two-dimensional semiconductor. Nat. Commun. 8, 15053 (2017).

11. $\mathrm{Wu}, \mathrm{W}$. et al. Locally defined quantum emission from epitaxial few-layer tungsten diselenide. Appl. Phys. Lett. 114, 213102-213105 (2019).

12. Xu, X., Yao, W., Xiao, D. \& Heinz, T. F. Spin and pseudospins in layered transition metal dichalcogenides. Nat. Phys. 10, 343-350 (2014).

13. Mak, K. F., He, K., Shan, J. \& Heinz, T. F. Control of valley polarization in monolayer $\mathrm{MoS}_{2}$ by optical helicity. Nat. Nanotechnol. 7, 494-498 (2012).

14. Chakraborty, C., Kinnischtzke, L., Goodfellow, K. M., Beams, R. \& Vamivakas, A. N. Voltage-controlled quantum light from an atomically thin semiconductor. Nat. Nanotechnol. 10, 507-511 (2015).

15. Srivastava, A. et al. Optically active quantum dots in monolayer $\mathrm{WSe}_{2}$. Nat. Nanotechnol. 10, 491-496 (2015).

16. Koperski, M. et al. Single photon emitters in exfoliated $\mathrm{WSe}_{2}$ structures. Nat. Nanotechnol. 10, 503-506 (2015).

17. He, Y.-M. et al. Single quantum emitters in monolayer semiconductors. Nat. Nanotechnol. 10, 497-502 (2015).

18. Sajid, A., Ford, M. J. \& Reimers, J. R. Single-photon emitters in hexagona boron nitride: a review of progress. Rep. Prog. Phys. 83, 044501 (2020).

19. Tran, T. T., Bray, K., Ford, M. J., Toth, M. \& Aharonovich, I. Quantum emission from hexagonal boron nitride monolayers. Nat. Nanotechnol. 11, 37-41 (2016).

20. Shepard, G. D. et al. Nanobubble induced formation of quantum emitters in monolayer semiconductors. 2D Mater. 4, 021019 (2017).

21. Koperski, M. et al. Optical properties of atomically thin transition metal dichalcogenides: observations and puzzles. Nanophotonics 6, 1289-1308 (2017).

22. Lezama, I. G. et al. Indirect-to-direct band gap crossover in few-layer MoTe2. Nano Lett. 15, 2336-2342 (2015).

23. Parto, K., Azzam, S. I., Banerjee, K. \& Moody, G. Defect and strain engineering of monolayer WSe2 enables site-controlled single-photon emission up to 150 K. Nat. Commun. 12, 1-8 (2021). 
24. Linhart, L. et al. Localized intervalley defect excitons as single-photon emitters in wse 2. Phys. Rev. Lett. 123, 146401 (2019).

25. Harats, M. G., Kirchhof, J. N., Qiao, M., Greben, K. \& Bolotin, K. I. Dynamics and efficient conversion of excitons to trions in non-uniformly strained monolayer $\mathrm{WS}_{2}$. Nat. Photonics 14, 324-329 (2020).

26. Chi, Z., Chen, H., Zhao, Q. \& Weng, Y.-X. Ultrafast carrier and phonon dynamics in few-layer 2H-MoTe2. J. Chem. Phys. 151, 114704 (2019).

27. Krishnamurthy, S. et al. PbS/CdS quantum dot room-temperature singleemitter spectroscopy reaches the telecom $\mathrm{O}$ and $\mathrm{S}$ bands via an engineered stability. ACS Nano 15, 575-587 (2020).

28. Strauf, S. et al. High-frequency single-photon source with polarization control. Nat. Photonics 1, 704-708 (2007).

29. Jeantet, A. et al. Widely tunable single-photon source from a carbon nanotube in the Purcell regime. Phys. Rev. Lett. 116, 247402 (2016).

30. Mangum, B. D., Ghosh, Y., Hollingsworth, J. A. \& Htoon, H. Disentangling the effects of clustering and multi-exciton emission in second-order photon correlation experiments. Opt. Express 21, 7419-7426 (2013).

31. Arora, A. et al. Valley Zeeman splitting and valley polarization of neutral and charged excitons in monolayer MoTe2 at high magnetic fields. Nano Lett. 16, 3624-3629 (2016).

32. Yu, L. et al. Site-controlled quantum emitters in monolayer MoSe2. Nano Lett. 21, 2376-2381 (2021).

33. He, Y.-M. et al. Cascaded emission of single photons from the biexciton in monolayered $\mathrm{WSe}_{2}$. Nat. Commun. 7, 13409 (2016).

34. Lu, X. et al. Optical initialization of a single spin-valley in charged $\mathrm{WSe}_{2}$ quantum dots. Nat. Nanotechnol. 14, 426-431 (2019).

35. Brotons-Gisbert, M. et al. Coulomb blockade in an atomically thin quantum dot coupled to a tunable Fermi reservoir. Nat. Nanotechnol. 14, 442-446 (2019).

36. Gammon, D., Snow, E. S., Shanabrook, B. V., Katzer, D. S. \& Park, D. Fine structure splitting in the optical spectra of single GaAs quantum dots. Phys. Rev. Lett. 76, 3005-3008 (1996).

37. Gammon, D., Snow, E. S., Shanabrook, B. V., Katzer, D. S. \& Park, D. Homogeneous linewidths in the optical spectrum of a single gallium arsenide quantum dot. Science 273, 87-90 (1996).

38. Jones, A. M. et al. Optical generation of excitonic valley coherence in monolayer $\mathrm{WSe}_{2}$. Nat. Nanotechnol. 8, 634-638 (2013).

39. Klein, J. et al. Site-selectively generated photon emitters in monolayer $\mathrm{MoS}_{2}$ via local helium ion irradiation. Nat. Commun. 10, 2755 (2019).

40. Luo, Y. et al. Carbon nanotube color centers in plasmonic nanocavities: a path to photon indistinguishability at telecom bands. Nano Lett. 19, 9037-9044 (2019).

\section{Acknowledgements}

We acknowledge the helpful discussion and technical support from Dr. Christopher Lane, Dr. Jianxin Zhu, Dr. Andrew Jones, and Mr. John Kevin Scott Baldwin. This work was performed at the Center for Integrated Nanotechnologies, an Office of Science User Facility operated for the U.S. Department of Energy (DOE) Office of Science. Los Alamos National Laboratory (LANL), an affirmative action equal opportunity employer, is managed by Triad National Security, LLC for the U.S. Department of Energy's NNSA, under contract $89233218 \mathrm{CNA} 000001$. Deterministic quantum emitter creation capability was developed under the support of DOE BES, QIS Infrastructure Development Program BES LANL22. H.Z., Y.Z., and H.H. acknowledge partial support form Laboratory Directed Research and Development (LDRD) program 20200104DR. H.H. is also partially supported by Quantum Science Center. M.T.P. is supported by LDRD 20190516ECR. H.Z. also acknowledge a partial support from LANL Director's Postdoctoral Fellow Award.

\section{Author contributions}

H.Z. and H.H. conceived the experiment. H.Z., under the supervision of H.H., primarily developed deterministic QE creation approach, designed and conducted the experiment, analyzed the data, and composed the paper. Y.Z. assisted in the experiment. M.T.P. assisted in the design of the nano-pillar samples and paper preparation.

\section{Competing interests}

The authors declare no competing interests.

\section{Additional information}

Supplementary information The online version contains supplementary material available at https://doi.org/10.1038/s41467-021-27033-w.

Correspondence and requests for materials should be addressed to Huan Zhao or Han Htoon.

Reprints and permission information is available at http://www.nature.com/reprints

Publisher's note Springer Nature remains neutral with regard to jurisdictional claims in published maps and institutional affiliations.Nature Communications thanks the anonymous reviewers for their contribution to the peer review of this work.

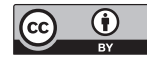

Open Access This article is licensed under a Creative Commons Attribution 4.0 International License, which permits use, sharing, adaptation, distribution and reproduction in any medium or format, as long as you give appropriate credit to the original author(s) and the source, provide a link to the Creative Commons license, and indicate if changes were made. The images or other third party material in this article are included in the article's Creative Commons license, unless indicated otherwise in a credit line to the material. If material is not included in the article's Creative Commons license and your intended use is not permitted by statutory regulation or exceeds the permitted use, you will need to obtain permission directly from the copyright holder. To view a copy of this license, visit http://creativecommons.org/ licenses/by/4.0/.

This is a U.S. Government work and not under copyright protection in the US; foreign copyright protection may apply 2021 